\title{
Bose-Einstein condensation of chromium
}

\author{
Axel Griesmaier * Jörg Werner, Sven Hensler, Jürgen Stuhler, and Tilman Pfau \\ 5. Physikalisches Institut, Universität Stuttgart, 70550 Stuttgart, Germany
}

(Dated: October 23, 2018)

\begin{abstract}
We report on the generation of a Bose-Einstein condensate in a gas of chromium atoms, which will make studies of the effects of anisotropic long-range interactions in degenerate quantum gases possible. The preparation of the chromium condensate requires novel cooling strategies that are adapted to its special electronic and magnetic properties. The final step to reach quantum degeneracy is forced evaporative cooling of ${ }^{52} \mathrm{Cr}$ atoms within a crossed optical dipole trap. At a critical temperature of $\mathrm{T}_{c} \approx 700 \mathrm{nK}$, we observe Bose-Einstein condensation by the appearance of a two-component velocity distribution. Released from an anisotropic trap, the condensate expands with an inversion of the aspect ratio. We observe critical behavior of the condensate fraction as a function of temperature and more than 50,000 condensed ${ }^{52} \mathrm{Cr}$ atoms.
\end{abstract}

PACS numbers: 03.75.Hh

Keywords: Bose-Einstein condesation, chromium, dipole-dipole interaction

The essential properties of degenerate quantum gases depend on range, strength and symmetry of the present interactions. Since the first observation of Bose-Einstein condensation in weakly interacting atomic gases, eight different elements have been Bose-Einstein condensed 1 , 2, 3, 4, 5, 6, 7, 8]. All these elements, mainly alkali atoms, interact dominantly via short-range isotropic potentials. Based on this effective contact interaction, many exciting phenomena have been studied [9, 10]. Examples are the realization of four-wave mixing with matter waves 11] as well as the observation of vortices 12, 13. and solitons 14, 15, 16] in degenerate quantum gases. Bose-Einstein condensates (BECs) with contact interaction have also been used to investigate solid-state physics problems like the Mott-metal-insulator transition 17, 18]. Tuning the contact interaction, the collapse and explosion ("Bosenova") of Bose-Einstein condensates has been studied [19] and new types of quantum matter like a Tonks-Girardeau gas have been realized [20].

In a chromium Bose-Einstein condensate, one can not only tune the short-range contact interaction using one of the recently observed Feshbach resonances 21] but also investigate effects of the longrange and anisotropic dipole-dipole interaction. This becomes possible because, compared to other Bose-condensed elements, the transition metal chromium has a unique electronic structure. The valence shell of its ground state contains six electrons with parallel spin alignment (electronic configuration: $\left.[\operatorname{Ar}] 3 d^{5} 4 s^{1}\right)$. For the bosonic chromium isotopes, which have no nuclear spin, this gives rise to a total electronic spin quantum number of 3 and a very high magnetic moment of $6 \mu_{\mathrm{B}}$ ( $\mu_{\mathrm{B}}$ is the Bohr magneton) in its ground state ${ }^{7} S_{3}$. Since the magnetic dipole-dipole interaction (MDDI) scales with the square of the magnetic moment, it is a factor of 36 higher for chromium than for alkali atoms. For this reason, dipole-dipole interactions which have not yet been investigated experimentally in degenerate quantum gases will become ob- servable in chromium BEC. For example, it was shown in 22. that the MDDI in chromium is strong enough to manifest itself in a well pronounced modification of the condensate expansion that depends on the orientation of the magnetic moments. Tuning the contact interaction between ${ }^{52} \mathrm{Cr}$ atoms close to zero will allow one to realize a dipolar BEC 23] in which the MDDI is the dominant interaction. This way, many predicted dipole-dipole phenomena, like the occurrence of a Maxon-Roton in the excitation spectrum of a dipolar BEC 24] or new kinds of quantum phase transitions [25, 26] as well as the stability and the ground state of dipolar BEC's 27, 28, 29] can be investigated experimentally. Since also the MDDI is tuneable [30], a degenerate quantum gas with adjustable long- and short-range interactions can be realized.

A chromium BEC is also unique with respect to technical applications of degenerate quantum gases. As a standard mask material in lithographic processes, chromium is a well suited element for atom lithography 31. It has already been used to grow nanostructures on substrates by direct deposition of laser-focused thermal atomic beams 32, 33]. Furthermore, structured doping has been demonstrated by simultaneously depositing a homogeneous matrix material and laser-focused chromium [34]. Performing the step from incoherent thermal atomic beams to coherent atom sources (BEC's), promises to increase the potential of atom lithography in a similar way like the invention of the laser did in classical optical lithography.

Our preparation scheme combines magneto-optical, magnetic and optical trapping techniques. It requires novel cooling strategies that are adapted to its special electronic and magnetic properties and to the need to circumvent relaxation processes originating from the dipolar character of the atoms. A beam of chromium atoms is generated by a high temperature effusion cell at $1600^{\circ} \mathrm{C}$ and slowed down by a Zeeman slower. Using a continuous loading scheme [35, 36] followed by an in-trap 


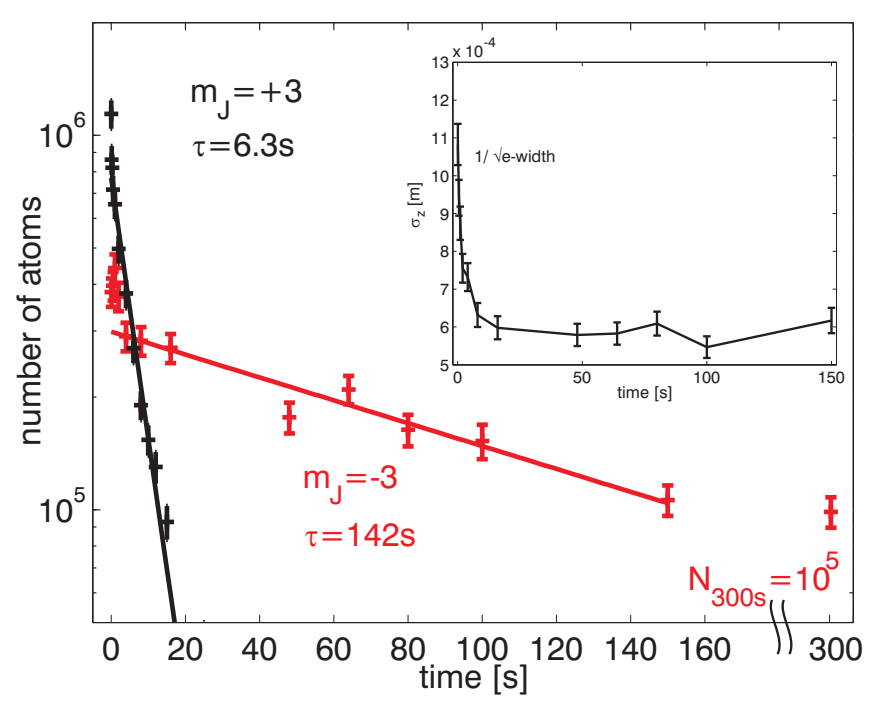

Figure 1: Comparison of trap lifetimes before (black) and after (red) pumping the atoms to the lowest Zeeman substate. The inset shows the change of the axial size of the expanded cloud in time of flight after different holding times.

Doppler-cooling stage [37], we prepare $\sim 1.3 \times 10^{8}$ atoms in the energetically highest projection $m_{J}=+3$ of the ${ }^{7} S_{3}$ ground state in a Ioffe-Pritchard trap. Subsequently, during $13 \mathrm{~s}$ of radiofrequency $(\mathrm{RF})$ induced evaporation, the atoms are cooled in the magnetic trap to a phase space density five orders of magnitude below from degeneracy. The extraordinary large magnetic dipole moment of chromium leads to increasing two-body loss in the form of dipolar relaxation 38 with increasing spacial density of the cloud. This causes RF-evaporation to become inefficient and prevents ${ }^{52} \mathrm{Cr}$ from reaching the regime of quantum degeneracy in a magnetic trap. To overcome this loss mechanism, the atoms have to be transferred into the energetically lowest Zeeman substate $m_{J}=-3$. In this state, energy conservation suppresses dipolar relaxation if the Zeeman splitting is much larger than the thermal energy of the atoms. As chromium atoms in states $m_{J}<0$ are repelled from regions with low magnetic fields, these atoms can not be trapped magnetically. We therefore adiabatically transfer the atoms into an optical dipole trap where the trapping forces are independent of the Zeeman substate. The optical trapping potential is formed by two beams produced by a $20 \mathrm{~W}$ fibre laser at $1064 \mathrm{~nm}$. The stronger horizontal trapping beam has a waist of $30 \mu \mathrm{m}$ and a power of up to $\sim 9 \mathrm{~W}$. The symmetry axis of the trapping potential formed by this beam coincides with the axis of our magnetic trap to achieve the largest overlap between the two trap volumes for efficient transfer into the optical trap. Ramping up this beam to its maximum intensity during the final step of the RF ramp, we are able to transfer $1.8 \times 10^{6}$ atoms into the optical trap.

The trap has a depth of $130 \mu \mathrm{K}$ and trap frequencies of $1450 \mathrm{~Hz}$ in radial and $12 \mathrm{~Hz}$ in axial direction. After switching off the magnetic trapping potential, we optically pump the atoms to the $m_{J}=-3$ state using a laser resonant to the ${ }^{7} S_{3} \rightarrow{ }^{7} P_{3}$ transition at an offset field of $9 \mathrm{G}$ in vertical direction. The efficiency of the transfer is close to $100 \%$ and is reflected in a dramatic increase of the lifetime of the trapped gas from $6 \mathrm{~s}$ in the $m_{J}=+3$ state to $>140 \mathrm{~s}$ in the $m_{J}=-3$ state as shown in figure 1 During all further steps of preparation, the offset field is kept on in order to prevent thermal redistribution among the other Zeeman states.

The optical transfer is followed by a $5 \mathrm{~s}$ stage of plain evaporation during which the number of atoms in the trap drops by $50 \%$ and the phase space density increases to $\sim 10^{-2}$. This effect becomes visible in a decrease of the axial size of the expanded cloud with increasing holding times in the trap (see inset of figure 11). To increase the local density and the elastic collision rate for evaporative cooling, a second beam in vertical direction with a waist of $50 \mu \mathrm{m}$ and a power of $\sim 4.5 \mathrm{~W}$ is additionally ramped up adiabatically within the first $5 \mathrm{~s}$ in the optical trap. After the intensity of the horizontal beam is reduced to $70 \%$ of its initial value within $10 \mathrm{~s}$, about 300,000 atoms are trapped in this crossed trap. Forced evaporation towards the critical temperature for the condensation proceeds now by gradually reducing the intensity of the horizontal beam within $11 \mathrm{~s}$. Degeneracy is reached at a remaining power of $\sim 800 \mathrm{~mW}$ in the horizontal beam. After holding the atoms in the final trapping potential for $25 \mathrm{~ms}$, we switch off both beams simultaneously and let the cloud expand freely for a variable time of flight. The cloud is then detected using a standard absorption imaging technique with a resonant probe beam propagating in the horizontal direction, perpendicular to both trapping beams. For the imaging, the magnetic field is rotated into the probe beam direction just before releasing the atoms from the trap.

The onset of quantum degeneracy becomes visible in absorption images of the relaxed chromium cloud. Figure 2 shows the profiles of the cloud after $5 \mathrm{~ms}$ of free expansion at different final powers of the horizontal trapping beam before releasing the cloud. Figure2(a) displays the situation at a final power of $1.4 \mathrm{~W}$. The cloud has the Gaussian profile of a pure thermal distribution corresponding to a temperature of $\sim 1.1 \mu \mathrm{K}$, very close to the critical temperature $T_{C}$. In (b), the cloud was released from a trap with a final power of $650 \mathrm{~mW}$ in the horizontal beam. The twocomponent distribution indicates the presence of a Bose-Einstein condensate. The temperature of the remaining thermal part of the cloud is $625 \mathrm{nK}$. Reducing the laser power even further to $\sim 370 \mathrm{~mW}$ leaves an almost pure condensate with more than 50,000 atoms and a non-Gaussian distribution as depicted in figure 2(c). At the end of our evaporation ramp, the trap frequencies in the visible axes are nearly equal. As expected, the expansion of the condensate is almost isotropic and the aspect 

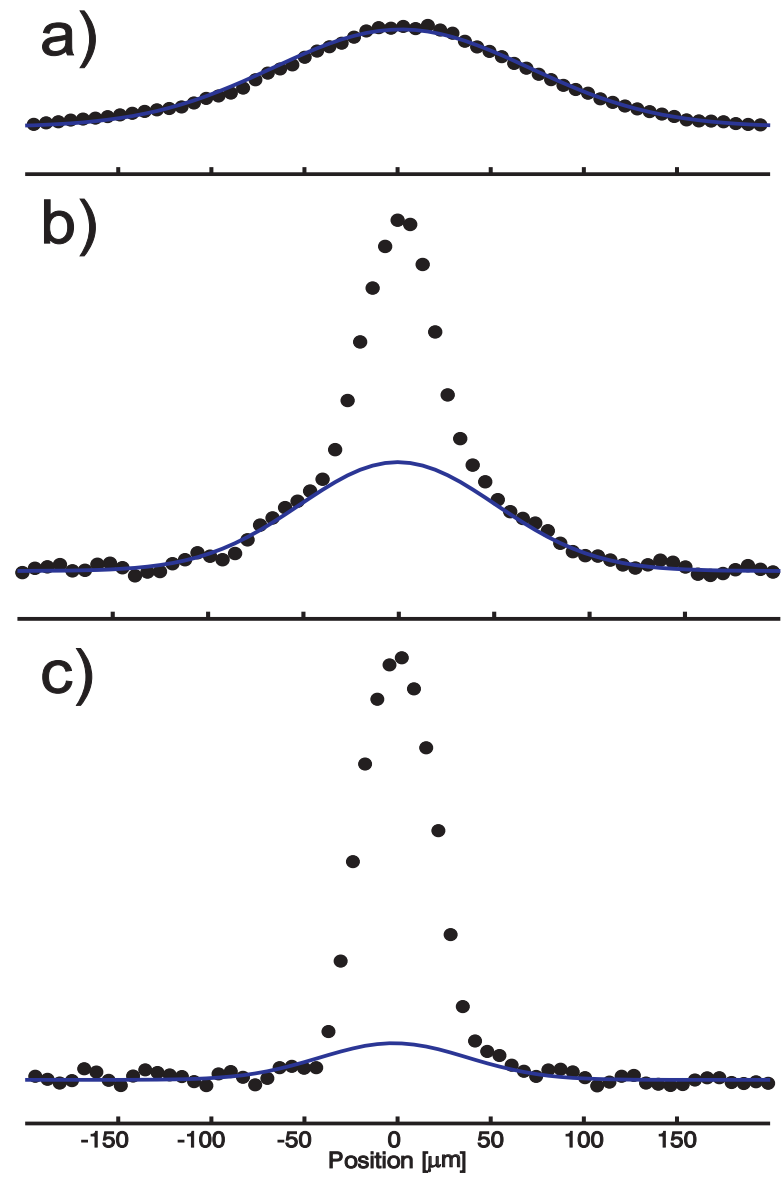

Figure 2: Density profiles from absorption images of atom clouds taken after $5 \mathrm{~ms}$ of ballistic expansion. A) thermal cloud at $1.1 \mu \mathrm{K}, \mathrm{B})$ two-component distribution at $625 \mathrm{nK}$, slightly below $T_{C}$, C) nearly pure condensate with 47,000 atoms.

ratio of the expanded condensate stays constant at $\sim 1$. A series of images of such a condensate taken after variable expansion times between $1 \mathrm{~ms}$ and $9 \mathrm{~ms}$ is shown in figure $3(a)$. The situation is different if the BEC is released from an anisotropic trapping potential. The images in figure 3(b) show the expansion of the condensate for the same time of flight series as before, but from a trap with a $\sim 20$ times higher intensity in the horizontal beam. In this case the BEC was prepared in the same way as in (a) except that the trapping potential was deformed by adiabatically increasing the horizontal laser power within $250 \mathrm{~ms}$ after the formation of the BEC. The series shows a clearly anisotropic expansion of the cloud. From a cigar shaped trap in the horizontal direction, the aspect ratio changes in time of flight to elongated in vertical direction. To show the critical behaviour at the point of emerging degeneracy, we determine the number of atoms in the condensate and the thermal fraction separately. We extract these numbers by fitting a two-component distribution function to the density profiles of the clouds at

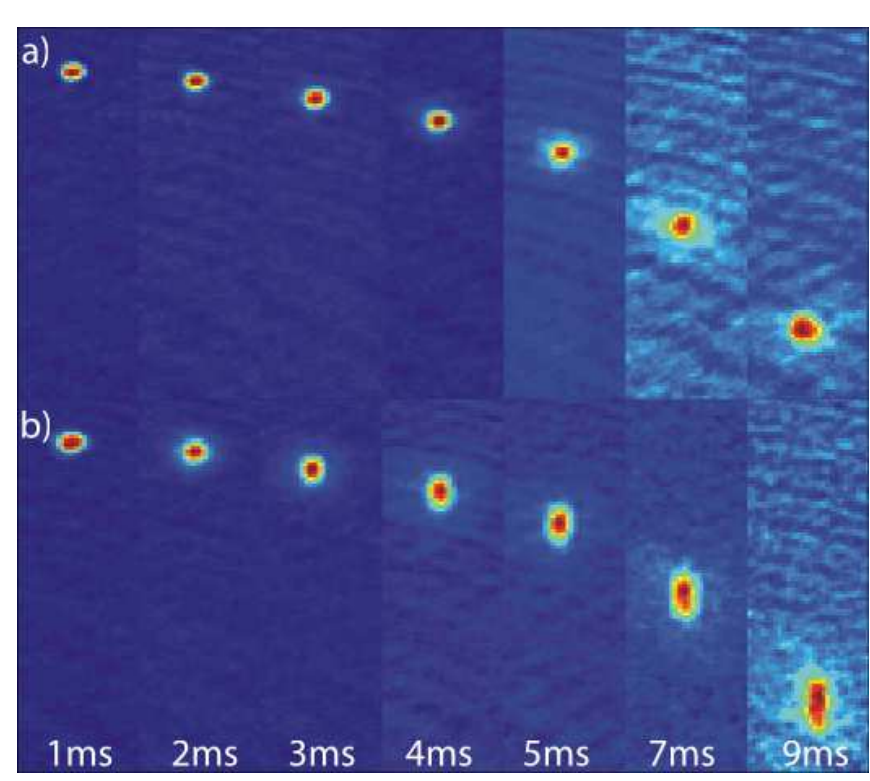

Figure 3: Time of flight series of absorption images with expansion times from $1 \mathrm{~ms}$ to $9 \mathrm{~ms}$. a) BEC released from an almost isotropic trap, b) BEC released from an anisotropic trap.

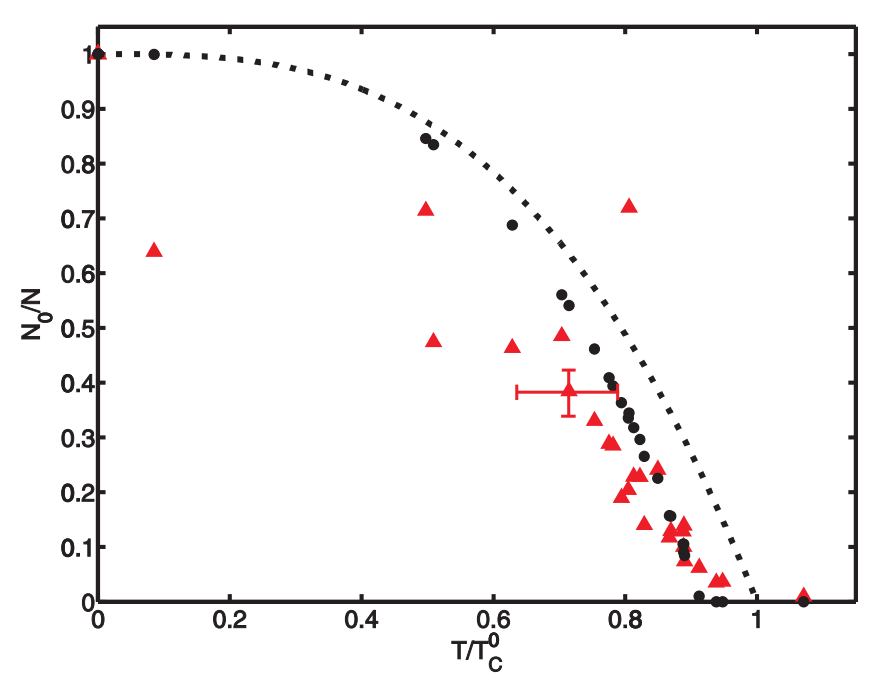

Figure 4: Condensate fraction $\left(N_{0} / N\right)$ dependence on temperature relative to the transition temperature of an ideal gas $\left(T / T_{C}^{0}\right)$ given by equation 1 Red triangles represent our measured data. Black circles represent the predicted fraction following equation 3 including corrections due to finite size effects and the contact interaction The dashed curve shows the dependence for the ideal gas. Error bars are mainly due to uncertainties in the measurement of the trap frequencies, temperature and number of atoms.

different final laser powers. In figure 4 the fraction of condensed atoms in the total number of atoms $\left(N_{0} / N\right)$ is plotted versus the ratio of the temperature of the remaining thermal part to the critical temperature $\left(T / T_{C}\right)$. When we approach the critical temperature from above $\left(T / T_{C}>1\right)$, the kink in the condensate fraction plot 
marks the onset of Bose-Einstein condensation and provides an experimental value for the critical temperature of $T_{\text {exp }} \sim 700 \mathrm{nK}$. Based on the trap frequencies, number of atoms and temperature, we have also calculated the expected condensate fraction for an ideal gas following the equation

$$
\frac{N_{0}}{N}=1-\left(\frac{T}{T_{C}}\right)^{3}
$$

with

$$
T_{C}^{0} \approx 0.94 \frac{\hbar \omega}{k_{B}} N^{1 / 3}
$$

being the critical temperature. When finite size effects as well as a correction arising from the contact interaction 39] are taken into account, the critical temperature is shifted to lower temperatures:

$$
T_{C}=T_{C}^{0}+\delta T_{C}^{i n t}+\delta T_{C}^{f s}
$$

where $\delta T_{C}^{f s}=-0.73 \frac{\bar{\omega}}{\omega} N^{-1 / 3} T_{C}^{0}$ is a shift in the critical temperature due to the finite number of atoms and $\delta T_{C}^{i n t}=-1.33 \frac{a}{a_{H O}} N^{1 / 6} T_{C}^{0}$ takes into account the contact interaction. Here $a=105 a_{0}$ is the chromium scattering length [21], $a_{0}$ being Bohr's radius, $a_{H O}$ is the harmonic oscillator length, $T$ is the temperature of the thermal cloud, $\omega$ is the geometric and $\bar{\omega}$ the arithmetic mean of the trap frequencies. These expected values are represented by black dots in figure 4 and demonstrate a good agreement of our data with the predicted dependence. In conclusion, we have demonstrated Bose-Einstein condensation of chromium atoms. We produce condensates with more than 50,000 chromium atoms, which is a very good basis for a series of promising experiments on the dipolar character of this novel quantum gas. We expect that both, long and short range interaction, can be tuned by magnetic fields. As chromium is a standard material in atom lithography, we also expect that an atom laser of chromium will have applications in lithography, possibly even enabling controlled deposition of single atoms.

We thank all members of our atom optics group for their encouragement and practical help. We acknowledge especially the contributions of Piet Schmidt and Axel Görlitz at earlier stages of the experiment. We thank Luis Santos, Paolo Pedri, Stefano Giovanazzi and Andrea Simoni for stimulating discussions. This work was supported by the SPP1116 of the German Science Foundation (DFG).
[2] K. B. Davis, M.-O. Mewes, M. R. Andrews, N. J. van Druten, D. S. Durfee, D. M. Kurn, and W. Ketterle, Phys. Rev. Lett. 75, 3969 (1995).

[3] C. C. Bradley, C. A. Sackett, J. J. Tollett, and R. G. Hulet, Phys. Rev. Lett. 75, 1687 (1995).

[4] D. G. Fried, T. C. Killian, L. Willmann, D. Landhuis, S. C. Moss, D. Kleppner, and T. J. Greytak, Phys. Rev. Lett. 81, 3811 (1998).

[5] G. Modugno, G. Ferrari, G. Roati, R. J. Brecha, A. Simoni, and M. Inguscio, Science 294, 1320 (2001).

[6] A. Robert, O. Sirjean, A. Browaeys, J. Poupard, S. Nowak, D. Boiron, and C. W. A. Aspect, Science 292, 461 (2001).

[7] T. Weber, J. Herbig, M. Mark, H.-C. Nägerl, and R. Grimm, Science 299, 232 (2003).

[8] Y. Takasu, K. Maki, K. Komori, T. Takano, K. Honda, M. Kumakura, T. Yabuzaki, and Y. Takahashi, Phys. Rev. Lett. 91, 040404 (2003).

[9] M. Weidemüller and C. Zimmermann, Interactions in Ultracold Gases (Wiley-VCH Verlag GmbH, 2003).

[10] J. R. Anglin and W. Ketterle, Nature 416, 211 (2002).

[11] L. Deng, E. W. Hagley, J. Wen, M. Trippenbach, Y. Band, P. S. Julienne, J. E. Simsarian, K. Helmerson, S. L. Rolston, and W. D. Phillips, Nature 398, 218 (1999).

[12] M. R. Matthews, B. P. Anderson, P. C. Haljan, D. S. Hall, C. E. Wieman, and E. A. Cornell, Phys. Rev. Lett. 83, 2498 (1999).

[13] K. W. Madison, F. Chevy, W. Wohlleben, and J. Dalibard, Phys. Rev. Lett. 84, 806 (2000).

[14] S. Burger, K. Bongs, S. Dettmer, W. Ertmer, K. Sengstock, A. Sanpera, G. V. Shlyapnikov, and M. Lewenstein, Phys. Rev. Lett. 83, 5198 (1999).

[15] J. Denschlag, J. E. Simsarian, D. L. Feder, C. W. Clark, L. A. Collins, J. Cubiziolles, L. Deng, E. W. Hagley, K. Helmerson, W. P. Reinhardt, et al., Science 287, 97 (2000).

[16] L. Khaykovich, F. Schreck, G. Ferrari, T. Bourdel, J. Cubizolles, L. Carr, Y.Castin, and C. Salomon, Science 296, 1290 (2002).

[17] M. Greiner, O. Mandel, T. Esslinger, T. Hänsch, and I. Bloch, Nature 415, 39 (2002).

[18] T. Stöferle, H. Moritz, C. Schori, M. Köhl, and T. Esslinger, Phys. Rev. Lett. 92, 130403 (2004).

[19] E. A. Donley, N. R. Claussen, S. L. Cornish, J. L. Roberts, E. A. Cornell, and C. E. Wieman, Nature 412, 295 (2001).

[20] B. Paredes, A. Widera, V. Murg, O. Mandel, S. Fölling, I. Cirac, G. V. Shlyapnikov, T. W. Hänsch, and I. Bloch, Nature 429, 277 (2004).

[21] J. Werner, A. Griesmaier, S. Hensler, A. Simoni, E. Tiesinga, J. Stuhler, and T. Pfau, Observation of feshbach resonances in an ultracold gas of ${ }^{52} \mathrm{cr}$, arXiv: condmat/0412049 (2004).

[22] S. Giovanazzi, A. Görlitz, and T. Pfau, J. Opt. B: Quantum Semiclass. Opt. 5, S208 (2003).

[23] M. Baranov, L. Dobrek, K. Góral, L. Santos, and M. Lewenstein, Physica Scripta T102, 74 (2002), and references therein.

[24] L. Santos, G. V. Shlyapnikov, and M. Lewenstein, Physical Review Letters 90, 250403 (2003).

* Electronic address: a.griesmaier@physik.uni-stuttgart.de

$\dagger$ URL:http://www.physik.uni-stuttgart.de/institute/pi/25

[1] M. H. Anderson, J. R. Ensher, M. R. Matthews, C. E. Wieman, and E. A. Cornell, Science 269, 198 (1995).
K. Góral, L. Santos, and M. Lewenstein, Phys. Rev. Lett. 88, 170406 (2002).

[26] S. Yi, L. You, and H. Pu, Phys. Rev. Lett. 93, 040403 
(2004).

[27] D. H. J. O'Dell, S. Giovanazzi, and C. Eberlein, Phys. Rev. Lett. 92, 250401 (2004).

[28] K. Góral and L. Santos, Phys. Rev. A 66, 023613 (2002).

[29] L. Santos, G. V. Shlyapnikov, P. Zoller, and M. Lewenstein, Phys. Rev. Lett. 85, 1791 (2000).

[30] S. Giovanazzi, A. Görlitz, and T. Pfau, Phys. Rev. Lett. 89, 130401 (2002).

[31] M. Oberthaler and T. Pfau, J. Phys.: Condens. Matter 15, R233 (2003).

[32] J. J. McClelland, R. E. Scholten, E. C. Palm, and R. J. Celotta, Science 262, 877 (1993).

[33] U. Drodofsky, J. Stuhler, T. Schulze, M. Drewsen, B. Brezger, T. Pfau, and J. Mlynek, Appl. Phys. B 65, 755 (1997).

[34] T. Schulze, T. Müther, D. Jürgens, B. Brezger, M. Oberthaler, B. Brezger, T. Pfau, and J. Mlynek,
Appl. Phys. Lett. 78, 12 (2001).

[35] P. O. Schmidt, S. Hensler, J. Werner, T. Binhammer, A. Görlitz, and T. Pfau, J. Opt. B: Quantum Semiclass. Opt. 5, S170 (2003).

[36] J. Stuhler, P. O. Schmidt, S. Hensler, J. Werner, J. Mlynek, and T. Pfau, Phys. Rev. A 64, 031405(R) (2001).

[37] P. O. Schmidt, S. Hensler, J. Werner, T. Binhammer, A. Görlitz, and T. Pfau, J. Opt. Soc. Am. B 20, 960 (2003).

[38] S. Hensler, J. Werner, A. Griesmaier, P. O. Schmidt, A. Görlitz, T. Pfau, and K. R. S. Giovanazzi, Appl. Phys. B 77, 765 (2003).

[39] S. Giorgini, L. P. Pitaevskii, and S. Stringari, Phys. Rev. A 54, R4633 (1996). 